\title{
Characterization of edge-colored complete graphs with properly colored Hamilton paths
}

\author{
Jinfeng Feng* $\quad$ Hans-Erik Giesen ${ }^{\dagger} \quad$ Yubao Guo ${ }^{\ddagger} \quad$ Gregory Gutin ${ }^{\S}$ \\ Tommy Jensen Arash Rafiey\|
}

\begin{abstract}
An edge-colored graph $H$ is properly colored if no two adjacent edges of $H$ have the same color. In 1997, J. Bang-Jensen and G. Gutin conjectured that an edgecolored complete graph $G$ has a properly colored Hamilton path if and only if $G$ has a spanning subgraph consisting of a properly colored path $C_{0}$ and a (possibly empty) collection of properly colored cycles $C_{1}, C_{2}, \ldots, C_{d}$ such that $V\left(C_{i}\right) \cap V\left(C_{j}\right)=\emptyset$ provided $0 \leq i<j \leq d$. We prove this conjecture.

Keywords: edge-colored graphs, properly colored Hamilton paths.
\end{abstract}

\section{Introduction}

Let $G=(V, E)$ be a complete graph, and let $c: E \rightarrow\{1,2, \ldots, \chi\}$ be a fixed (not necessarily proper) edge-coloring of $G$ with $\chi$ colors, $\chi \geq 2$. With given $c, G$ is called a $\chi$-edge-colored (or, edge-colored) complete graph. A subgraph $H \subseteq G$ is called properly colored if $c$ defines a proper edge-coloring of $H$.

The existence of properly colored Hamilton paths and cycles has been studied in several papers; this topic was surveyed in [2] and later in Chapter 11 of [3]. While there are characterizations $[6,11]$ (see also Chapter 11 of [3]) of 2-edge-colored complete graphs with properly colored Hamilton cycles, no such characterization is known for $\chi$-edgecolored complete graphs with $\chi \geq 3$, and it is still an open question to determine the computational complexity of this problem [8].

\footnotetext{
${ }^{*}$ Lehrstuhl C fuer Mathematik, RWTH Aachen University, 52056 Aachen, Germany.

${ }^{\dagger}$ Lehrstuhl C fuer Mathematik, RWTH Aachen University, 52056 Aachen, Germany.

${ }^{\ddagger}$ Lehrstuhl C fuer Mathematik, RWTH Aachen University, 52056 Aachen, Germany.

${ }^{\S}$ Corresponding author. Department of Computer Science, Royal Holloway, University of London, Egham, Surrey, TW20 0EX, UK. Gutin@cs.rhul.ac.uk

『Institut fuer Mathematik, Universitat Klagenfurt, Universitatsstrasse 65-67, 9020 Klagenfurt, Austria.

"Department of Computer Science, Royal Holloway, University of London, Egham, Surrey, TW20 0EX, UK.
} 
The most studied possibly sufficient condition for an edge-colored complete graph with $n$ vertices to have a properly colored Hamilton cycle is $\Delta_{m o n}<\lfloor n / 2\rfloor$, where $\Delta_{m o n}$ is the maximal number of edges of the same color incident to the same vertex. This was conjectured by B. Bollobás and P. Erdős [9] in 1976, but remains unsolved. The best result so far for 'small' values of $n$ is by J. Shearer [12]: $7 \Delta_{m o n}<\lfloor n / 2\rfloor$ guarantees the existence of a properly colored Hamilton cycle. The best result so far for large values of $n$ is due to N. Alon and G. Gutin [1]: For every $\epsilon>0$ and $n=n_{\epsilon}$ large enough, $\Delta_{\text {mon }} \leq\left(1-\frac{1}{\sqrt{2}}-\epsilon\right)\lfloor n / 2\rfloor$ implies the existence of a properly colored Hamilton cycle.

For the case of properly colored Hamilton paths, the situation is somewhat different. Let the abbreviation PCHP stand for "properly colored Hamilton path". Let $G$ be an edge-colored graph. A properly colored cycle factor of $G$ is a spanning subgraph of $G$ consisting of properly colored cycles $C_{1}, C_{2}, \ldots, C_{d}$ such that $V\left(C_{i}\right) \cap V\left(C_{j}\right)=\emptyset$ provided $1 \leq i<j \leq d$. A properly colored 1-path-cycle factor of $G$ is a spanning subgraph of $G$ consisting of a properly colored path $C_{0}$ and a (possibly empty) collection of properly colored cycles $C_{1}, C_{2}, \ldots, C_{d}$ such that $V\left(C_{i}\right) \cap V\left(C_{j}\right)=\emptyset$ provided $0 \leq i<j \leq d$.

The following theorem gives a PCHP characterization for the case of just two colors:

Theorem 1.1 [2] A 2-edge-colored complete graph $G$ has a PCHP if and only if $G$ contains a properly colored 1-path-cycle factor.

It is conjectured in [2] that the above theorem holds for any number of colors. We call it the BJG conjecture. In support of the BJG conjecture, the following result was proved in [5]: If a $\chi$-edge-colored complete graph $G(\chi \geq 2)$ contains a properly colored cycle factor, then $G$ contains a PCHP.

It is easy to see that the BJG conjecture in [2] can be reduced to the following:

Conjecture 1.2 (PCHP Conjecture) Let $\chi \geq 3$ and let $G$ be a $\chi$-edge-colored complete graph. Assume that there exist $C, P \subseteq G$, where $C$ is a properly colored cycle and $P$ a properly colored path, such that $V(C) \cap V(P)=\emptyset$ and $V(C) \cup V(P)=V(G)$. Then $G$ contains a PCHP.

In this paper we prove the PCHP conjecture and, thus, the BJG conjecture. Since it takes polynomial time to check whether an edge-colored graph has a properly colored 1-path-cycle factor [2], our result implies that the PCHP problem is polynomial time solvable for edge-colored complete graphs. The proof of Theorem 2.1 is constructive and can be turned into a polynomial time algorithm for transforming a a properly colored 1-path-cycle factor into a properly colored Hamilton path.

This gives, in particular, some indication that the problem of the existence of a properly colored Hamilton cycle in an edge-colored graph may be polynomial time solvable after all. 
The situation may remind one of that with the existence of Hamilton paths and cycles in semicomplete multipartite digraphs (SMDs) [4] (see also Chapter 5 in [3]). Both Hamilton path and cycle problems for SMDs are polynomial time solvable, but only for the Hamilton path problem we have a nice characterization (see, e.g., [10] or Chapter 5 in [3]) so far.

In passing we mention a simple sufficient condition proved in [7] for the existence of a PCHP in an edge-colored $K_{n}: K_{n}$ has no monochromatic triangles.

\section{Results}

If $H$ is connected, the distance in $H$ between two vertices $u, v \in V(H)$ is the length of a shortest path in $H$ from $u$ to $v$, and we denote it by $\operatorname{dist}_{H}(u, v)$.

Theorem 2.1 The PCHP Conjecture holds.

Proof: Let $C=v_{1} \ldots v_{n} v_{1}(n \geq 3)$ and $P=u_{1} \ldots u_{m}(m \geq 1)$. Throughout we will perform addition and subtraction in the indices of the vertices $v_{j} \in C$ modulo $n$.

Let $j \in\{1,2, \ldots, n\}$. If $m \geq 2$ and $c\left(u_{1} v_{j}\right) \neq c\left(u_{1} u_{2}\right)$, then at least one of the paths $u_{m} u_{m-1} \ldots u_{1} v_{j} v_{j+1} \ldots v_{j-1}$ and $u_{m} u_{m-1} \ldots u_{1} v_{j} v_{j-1} \ldots v_{j+1}$ is a PCHP. Similarly there exists a PCHP if $c\left(u_{m} v_{j}\right) \neq c\left(u_{m-1} u_{m-2}\right)$. So we may assume the following:

(1) If $m \geq 2$, then $c\left(u_{1} v_{j}\right)=c\left(u_{1} u_{2}\right)$ and $c\left(u_{m} v_{j}\right)=c\left(u_{m-1} u_{m}\right)$ for every $j=$ $1,2, \ldots, n$.

Thus, to complete the proof of this theorem it suffices to prove the following claim:

Claim A If (1) is satisfied, then there exists a PCHP $H$ in $G$ with $u_{1}$ as its first vertex, such that the initial edge of $H$ is either $u_{1} u_{2}$ or one of the edges $u_{1} v_{j}(1 \leq j \leq n)$, and such that if $m \geq 2$, then $u_{m}$ is the last vertex of $H$ and the last edge of $H$ is either $u_{m-1} u_{m}$ or one of the edges $v_{j} u_{m}(1 \leq j \leq n)$.

Let $b(P, C)=2(n-3)+m$; we notice that $b(P, C) \geq 1$. Suppose that Claim A is false, and let $(G, P, C, c)$ be a counterexample with a minimal value of $b(P, C)$.

If $m=1$, then either $u_{1} v_{1} v_{2} \ldots v_{n}$ or $u_{1} v_{1} v_{n} \ldots v_{2}$ is a PCHP as desired. Thus, we have established $m \geq 2$ and $b(P, C) \geq 2$.

Now we prove

(2) $m \geq 3$. 
Suppose that $m=2$. With $x=c\left(u_{1} u_{2}\right)$, we have $c\left(u_{1} v_{j}\right)=c\left(u_{2} v_{j}\right)=x$ for all $j=$ $1,2, \ldots, n$, by (1). Choose $r$ such that $c\left(v_{r-1} v_{r}\right) \neq x \neq c\left(v_{r+1} v_{r+2}\right)$; this is possible if $r$ can be chosen with $c\left(v_{r} v_{r+1}\right)=x$, and otherwise it is trivial. But then the path $u_{1} v_{r} v_{r-1} \ldots v_{r+2} v_{r+1} u_{2}$ yields a contradiction.

We continue to prove further properties of the coloring $c$.

(3) For every $i, 1<i<m$, there exist $r$ and $s(1 \leq r, s \leq n)$, such that $c\left(u_{i} u_{i+1}\right) \neq$ $c\left(u_{i} v_{r}\right), c\left(u_{i-1} u_{i}\right) \neq c\left(u_{i} v_{s}\right)$.

Otherwise the path $P^{\prime}=P-u_{1}-u_{2}-\cdots-u_{i-1}$ satisfies (1) in $G^{\prime}=G-u_{1}-u_{2}-\cdots-u_{i-1}$ (with $u_{i}$ in place of $u_{1}$ ). Since $b\left(P^{\prime}, C\right)<b(P, C)$, there exists a PCHP $H^{\prime}$ in $G^{\prime}$ that starts from $u_{i}$ with one of the edges $u_{i} u_{i+1}$ or $u_{i} v_{j}, 1 \leq j \leq n$ and finishes in $u_{m}$ with $u_{m-1} u_{m}$ or with an edge $v_{k} u_{m}, 1 \leq k \leq n$. Then $u_{1} \ldots u_{i-1} H^{\prime}$ is a PCHP in $G$ of the desired type having $u_{1} u_{2}$ as its initial edge, a contradiction. A similar argument shows the existence of $s$.

Suppose that $c\left(v_{i-1} v_{i}\right)=c\left(v_{j} v_{j+1}\right)=x$, where $\operatorname{dist}_{C}\left(v_{i}, v_{j+1}\right) \geq 3$. If $c\left(v_{i} v_{j}\right)=x$, then define $G^{\prime}=G-v_{i}-v_{i+1}-\cdots-v_{j}$ and define $c^{\prime}: E\left(G^{\prime}\right) \rightarrow\{1,2, \ldots, \chi\}$ by

$$
c^{\prime}(e)=\left\{\begin{array}{cl}
x & \text { if } e=v_{i-1} v_{j+1} \\
c(e) & \text { otherwise }
\end{array}\right.
$$

Both $P$ and the cycle $C^{\prime}=v_{j+1} v_{j+2} \ldots v_{n} v_{1} \ldots v_{i-1} v_{j+1}$ are properly colored by $c^{\prime}$. Moreover, $P$ clearly satisfies (1) with respect to $C^{\prime}$. Since $b\left(P, C^{\prime}\right)<b(P, C)$, there exists a PCHP $P_{1}$ in $G^{\prime}$ with initial edge $u_{1} u_{2}$ or $u_{1} v_{r}$ for some $r \in\{j+1, j+2, \ldots, n, 1, \ldots, i-1\}$. If $v_{i-1} v_{j+1} \in P_{1}$, then we find the desired PCHP $H$ in $G$ by replacing the edge $v_{i-1} v_{j+1}$ by the path $v_{i-1} v_{i} \ldots v_{j} v_{j+1}$. Otherwise, if $v_{i-1} v_{j+1} \notin P_{1}$, then $P_{1}$ is properly colored in $G$ and satisfies (1) with respect to $C^{\prime \prime}=v_{i} v_{i+1} \ldots v_{j} v_{i}$, which is a properly colored cycle. By $b\left(P_{1}, C^{\prime \prime}\right)<b(P, C)$, the desired PCHP exists in $G$, a contradiction.

Thus, we have the following:

(4) Assume that $\operatorname{dist}_{C}\left(v_{s}, v_{t+1}\right) \geq 3$. If $c\left(v_{s-1} v_{s}\right)=c\left(v_{t} v_{t+1}\right)=x$, then $c\left(v_{s} v_{t}\right) \neq x$.

Consider the path $u_{1} \ldots u_{p-2} u_{p-1} v_{q-1} v_{q-2} \ldots v_{q+1} v_{q} u_{p} u_{p+1} \ldots u_{m}$. As it cannot be a PCHP we conclude the following.

(5) Let $2 \leq p \leq m$ and $1 \leq q \leq n$. Then at least one of the following holds. 


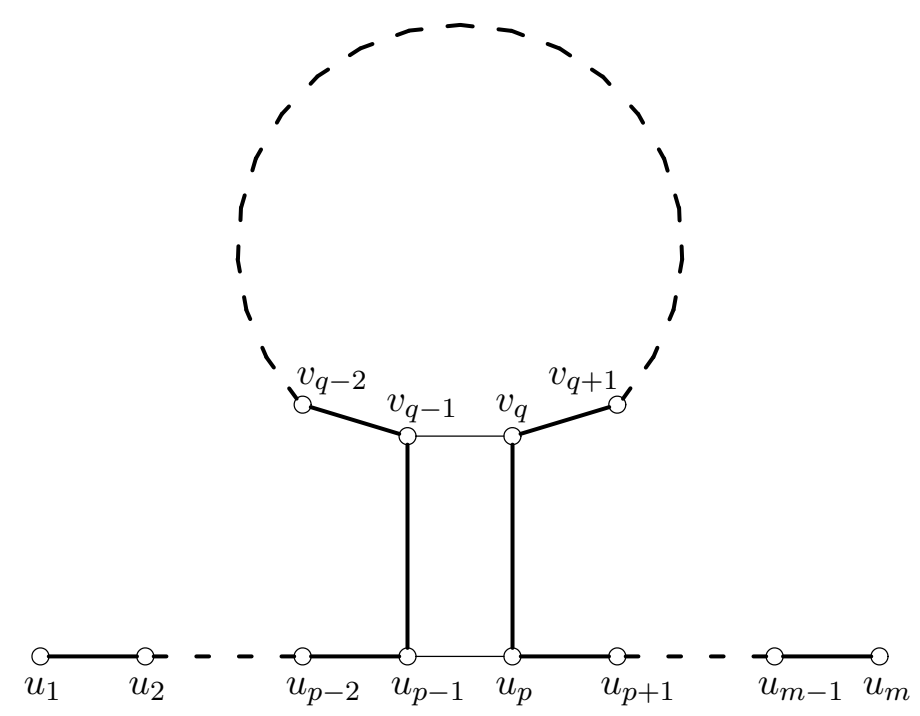

Figure 1: The path used to prove (5).
a) $p \geq 3$ and $c\left(u_{p-2} u_{p-1}\right)=c\left(u_{p-1} v_{q-1}\right)$
b) $c\left(u_{p-1} v_{q-1}\right)=c\left(v_{q-2} v_{q-1}\right)$
c) $c\left(u_{p} v_{q}\right)=c\left(v_{q} v_{q+1}\right)$
d) $p<m$ and $c\left(u_{p} v_{q}\right)=c\left(u_{p} u_{p+1}\right)$.

Considering the path $u_{1} \ldots u_{p-2} u_{p-1} v_{q+1} v_{q+2} \ldots v_{q-1} v_{q} u_{p} u_{p+1} \ldots u_{m}$ similarly leads to:

(6) Let $2 \leq p \leq m$ and $1 \leq q \leq n$. Then at least one of the following holds.
a) $p \geq 3$ and $c\left(u_{p-2} u_{p-1}\right)=c\left(u_{p-1} v_{q+1}\right)$
b) $c\left(u_{p-1} v_{q+1}\right)=c\left(v_{q+1} v_{q+2}\right)$
c) $c\left(u_{p} v_{q}\right)=c\left(v_{q-1} v_{q}\right)$
d) $p<m$ and $c\left(u_{p} v_{q}\right)=c\left(u_{p} u_{p+1}\right)$.

In several of the following applications of (5) and (6) it will be useful to note that (5c) and (6c) are mutually exclusive statements for any values of $p$ and $q$, since $C$ is properly colored, and that $(5 \mathrm{~d})$ and $(6 \mathrm{~d})$ are identical statements.

For the remaining part of the paper we define $x=c\left(u_{1} u_{2}\right)$ and $y=c\left(u_{m-1} u_{m}\right)$.

(7) Assume $c\left(u_{2} v_{j}\right)=z \neq c\left(u_{2} u_{3}\right)$ for some $j \in\{1,2, \ldots, n\}$. 


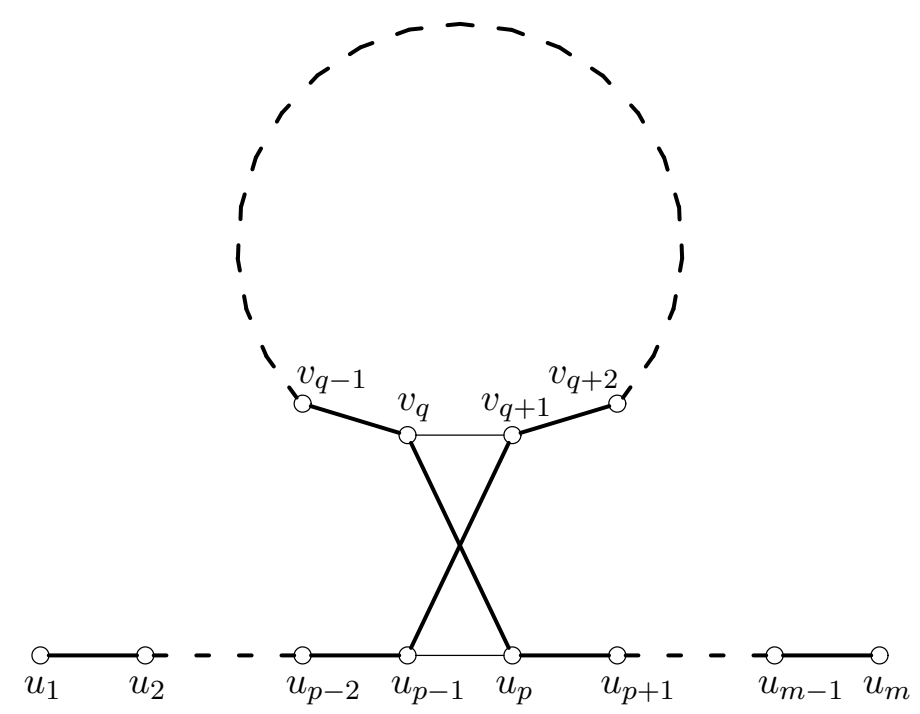

Figure 2: The path used to prove (6).

a) If $c\left(v_{j} v_{j+1}\right) \neq z$ then $c\left(v_{j-2} v_{j-1}\right)=x$, and if $c\left(v_{j-1} v_{j}\right) \neq z$ then $c\left(v_{j+1} v_{j+2}\right)=x$.

b) If $z=x$ or $c\left(v_{j-1} v_{j}\right) \neq z \neq c\left(v_{j} v_{j+1}\right)$, then $c\left(v_{j-2} v_{j-1}\right)=c\left(v_{j+1} v_{j+2}\right)=$ $c\left(v_{j-1} v_{j+1}\right)=x$ and $n \in\{3,5\}$.

If $c\left(v_{j-1} v_{j}\right) \neq z$, then $c\left(v_{j+1} v_{j+2}\right)=x$ follows from (6) with $p=2$ and $q=j$ (only (b) of (6) is not necessarily false). If $c\left(v_{j} v_{j+1}\right) \neq z$, then $c\left(v_{j-2} v_{j-1}\right)=x$ similarly follows from (5). This shows (a).

Now assume $c\left(v_{j-1} v_{j}\right) \neq z \neq c\left(v_{j} v_{j+1}\right)$ or $z=x$. In the case $z=x$ the fact that $c$ is a proper coloring of $C$ together with (a) implies $c\left(v_{j-2} v_{j-1}\right)=c\left(v_{j+1} v_{j+2}\right)=x$. The same conclusion follows directly from (a) when $c\left(v_{j-1} v_{j}\right) \neq z \neq c\left(v_{j} v_{j+1}\right)$. By symmetry, and since $c\left(v_{j-1} v_{j}\right) \neq c\left(v_{j} v_{j+1}\right)$, we may assume $c\left(v_{j-1} v_{j+1}\right) \neq c\left(v_{j} v_{j+1}\right)$. Since the Hamilton path $u_{1} v_{j+2} v_{j+3} \ldots v_{j-2} v_{j-1} v_{j+1} v_{j} u_{2} \ldots u_{m}$ fails to be a PCHP, it follows that $c\left(v_{j-1} v_{j+1}\right)=x$.

Consider (4) for $s=j-1$ and $t=j+1$. The conclusion $c\left(v_{s} v_{t}\right) \neq x$ of (4) implies $\operatorname{dist}_{C}\left(v_{j-1}, v_{j+2}\right) \leq 2$, which is only possible for $n \leq 5$. Moreover, the edges $v_{j-2} v_{j-1}$ and $v_{j+1} v_{j+2}$, both of color $x$, are not adjacent on $C$, which implies $n \neq 4$. Thus (b) is proved.

(8) Assume $c\left(u_{m-1} v_{k}\right)=z \neq c\left(u_{m-2} u_{m-1}\right)$ for some $k \in\{1,2, \ldots, n\}$.

a) If $c\left(v_{k-1} v_{k}\right) \neq z$ then $c\left(v_{k+1} v_{k+2}\right)=y$, and if $c\left(v_{k} v_{k+1}\right) \neq z$ then $c\left(v_{k-2} v_{k-1}\right)=y$.

b) If $z=y$ or $c\left(v_{k-1} v_{k}\right) \neq z \neq c\left(v_{k} v_{k+1}\right)$, then $c\left(v_{k-2} v_{k-1}\right)=c\left(v_{k+1} v_{k+2}\right)=$ $c\left(v_{k-1} v_{k+1}\right)=y$ and $n \in\{3,5\}$. 
The proof of (8) is similar to that of (7).

(9) Assume $c\left(u_{2} v_{j}\right) \neq x$ for all $j=1,2, \ldots, n$. Then there exists $j \in\{1,2, \ldots, n\}$ such that

a) $c\left(u_{2} v_{j-1}\right)=c\left(u_{2} v_{j}\right) \neq c\left(u_{2} u_{3}\right)$, and

b) $c\left(v_{j-2} v_{j-1}\right)=c\left(v_{j} v_{j+1}\right)=x$.

By (3) there exists $j \in\{1,2, \ldots, n\}$ such that $c\left(u_{2} v_{j}\right)=z \neq c\left(u_{2} u_{3}\right)$. We may assume $c\left(v_{j} v_{j+1}\right) \neq z$ (if not, then $c\left(v_{j-1} v_{j}\right) \neq z$ holds, and we may renumber the vertices on $C$ so that $v_{j+\ell}$ becomes $v_{j-\ell}$ for all $\ell=0,1,2, \ldots$ without change of the conclusion). Then (7a) implies $c\left(v_{j-2} v_{j-1}\right)=x$.

Again by (3) there exists $r \in\{1,2, \ldots, n\}$ such that if $m \geq 4$, then $c\left(u_{3} v_{r}\right) \neq c\left(u_{3} u_{4}\right)$ holds.

Suppose $c\left(u_{2} v_{j-1}\right) \neq z$. Since $u_{1} v_{r+1} v_{r+2} \ldots v_{j-2} v_{j-1} u_{2} v_{j} v_{j+1} \ldots v_{r-1} v_{r} u_{3} u_{4} \ldots u_{m}$ is not a PCHP, at least one of the following holds:

i) $c\left(u_{1} v_{r+1}\right)=c\left(v_{r+1} v_{r+2}\right)$,

ii) $c\left(u_{3} v_{r}\right)=c\left(v_{r-1} v_{r}\right)$,

iii) $r=j$ and $c\left(u_{2} v_{r}\right)=c\left(u_{3} v_{r}\right)$.

Since $u_{1} v_{r-1} v_{r-2} \ldots v_{j+1} v_{j} u_{2} v_{j-1} v_{j-2} \ldots v_{r+1} v_{r} u_{3} u_{4} \ldots u_{m}$ is not a PCHP, at least one of the following holds:

iv) $c\left(u_{1} v_{r-1}\right)=c\left(v_{r-2} v_{r-1}\right)$,

v) $c\left(u_{3} v_{r}\right)=c\left(v_{r} v_{r+1}\right)$,

vi) $r=j-1$ and $c\left(u_{2} v_{r}\right)=c\left(u_{3} v_{r}\right)$.

Let $p=3$ and $q=r$, and observe that neither of (5a), (5d), (6a) or (6d) holds.

We will now show that (iii) and (vi) do not hold. If $r=j$, then $c\left(v_{r-2} v_{r-1}\right)=x \neq$ $c\left(u_{2} v_{r-1}\right)$, hence also (5b) does not hold, and (5c) must be satisfied, that is, $c\left(u_{3} v_{r}\right)=$ $c\left(v_{r} v_{r+1}\right)$. In particular, if $r=j$, then $c\left(u_{3} v_{r}\right)=c\left(v_{j} v_{j+1}\right) \neq z=c\left(u_{2} v_{j}\right)$, contrary to (iii).

If $r=j-1$, then $c\left(u_{2} v_{r+1}\right)=c\left(u_{2} v_{j}\right)=z$ and $c\left(v_{r+1} v_{r+2}\right)=c\left(v_{j} v_{j+1}\right) \neq z$, so $(6 \mathrm{~b})$ does not hold. Then $(6 \mathrm{c})$ implies $c\left(u_{3} v_{r}\right)=c\left(v_{r-1} v_{r}\right)=c\left(v_{j-2} v_{j-1}\right)=x$. In particular, if $r=j-1$, then $c\left(u_{2} v_{r}\right) \neq c\left(u_{3} v_{r}\right)$ follows, hence also (vi) does not hold. 
We deduce that (i) or (ii) is true, and that (iv) or (v) is true. Now (6c) is equivalent to (ii), and (6b) and (i) are not both true (by (1) and our assumption), therefore (ii) holds. Similarly (5c) is equivalent to (v), and (5b) contradicts (iv), so also (v) holds. But (ii) contradicts (v), since $C$ is properly colored. This establishes $c\left(u_{2} v_{j-1}\right)=z$.

Finally $c\left(v_{j} v_{j+1}\right)=x$ follows from (7a).

(10) Assume $c\left(u_{m-1} v_{j}\right) \neq y$ for all $j=1,2, \ldots, n$. Then there exists $k \in\{1,2, \ldots, n\}$ such that

a) $c\left(u_{m-1} v_{k-1}\right)=c\left(u_{m-1} v_{k}\right) \neq c\left(u_{m-2} u_{m-1}\right)$, and

b) $c\left(v_{k-2} v_{k-1}\right)=c\left(v_{k} v_{k+1}\right)=y$.

The proof is similar to the proof of (9).

(11) Assume $z=c\left(u_{2} v_{j}\right)=c\left(u_{2} v_{j-1}\right) \notin\left\{x, c\left(u_{2} u_{3}\right)\right\}$ for some $j \in\{1,2, \ldots, n\}$. Furthermore assume $c\left(v_{j} v_{j+1}\right) \neq z$. Then one of the following holds:

a) $n$ is an even number, and $n / 2$ edges of $C$ have color $x$.

b) If $c\left(v_{j^{\prime}} v_{j^{\prime}+1}\right)=x$, then $v_{j^{\prime}} \in S=\left\{v_{j-4}, v_{j-2}, v_{j}, v_{j+2}\right\}$.

First $c\left(v_{j-2} v_{j-1}\right)=c\left(v_{j} v_{j+1}\right)=x$ follows from two applications of (7a), applying (7) to $u_{2} v_{j}$ and $u_{2} v_{j-1}$ in this order. Since $C$ is properly colored, we conclude that $c\left(v_{j^{\prime}} v_{j^{\prime}+1}\right)=x$ implies $v_{j^{\prime}} \notin\left\{v_{j-3}, v_{j-1}, v_{j+1}\right\}$. Assume that (b) does not hold, and choose any $v_{j^{\prime}} \in$ $V(C) \backslash S$ satisfying $c\left(v_{j^{\prime}} v_{j^{\prime}+1}\right)=x$. Then $n \geq 8$ follows from $v_{j^{\prime}} \notin S \cup\left\{v_{j-3}, v_{j-1}, v_{j+1}\right\}$. We will prove the following statement.

$(*) c\left(v_{j^{\prime}-2} v_{j^{\prime}-1}\right)=c\left(v_{j^{\prime}+2} v_{j^{\prime}+3}\right)=x$.

First we suppose $c\left(v_{j^{\prime}-2} v_{j^{\prime}-1}\right) \neq x$. Then $v_{j^{\prime}} \notin S \cup\left\{v_{j-3}, v_{j-1}, v_{j+1}\right\}$ and $n \geq 8$ imply $\operatorname{dist}_{C}\left(v_{j-1}, v_{j^{\prime}+1}\right) \geq 3$. By (4) with $s=j-1$ and $t=j^{\prime}$ we have $c\left(v_{j-1} v_{j^{\prime}}\right) \neq$ $x$. We consider the path $P_{1}=u_{1} v_{j^{\prime}-1} v_{j^{\prime}-2} \ldots v_{j+1} v_{j} u_{2} u_{3} \ldots u_{m}$ and the cycle $C_{1}=$ $v_{j^{\prime}} v_{j^{\prime}+1} \ldots v_{j-2} v_{j-1} v_{j^{\prime}}$, which are properly colored and satisfy (1). Since $b\left(C_{1}, P_{1}\right)<$ $b(C, P)$ holds, our minimality assumption yields a PCHP as in Claim A, which is a contradiction. So $c\left(v_{j^{\prime}-2} v_{j^{\prime}-1}\right)=x$ holds. Now suppose $c\left(v_{j^{\prime}+2} v_{j^{\prime}+3}\right) \neq x$. Then by $(4)$ with $s=j$ and $t=j^{\prime}+1$ we similarly have $c\left(v_{j} v_{j^{\prime}+1}\right) \neq x$, and we consider the path $P_{2}=u_{1} v_{j^{\prime}+2} v_{j^{\prime}+3} \ldots v_{j-2} v_{j-1} u_{2} u_{3} \ldots u_{m}$ and the cycle $C_{2}=v_{j^{\prime}+1} v_{j^{\prime}} \ldots v_{j+1} v_{j} v_{j^{\prime}+1}$ instead, again with a contradiction. Thus we have also $c\left(v_{j^{\prime}+2} v_{j^{\prime}+3}\right)=x$, which finishes the proof of $(*)$. 
Applying $(*)$ recursively, it follows that $c\left(v_{j^{\prime}+2 \ell} v_{j^{\prime}+2 \ell+1}\right)=x$ holds for every $\ell \in \mathbb{N}$ with $v_{j^{\prime}+2 \ell} \notin S \cup\left\{v_{j-3}, v_{j-1}, v_{j+1}\right\}$. In particular, either $c\left(v_{j+3} v_{j+4}\right)=x$ or $c\left(v_{j+4} v_{j+5}\right)=x$ holds $\left(v_{j+3} \notin S\right.$ follows from $n \geq 8$, and $v_{j+4} \in S$ only occurs if $n=8$ and $v_{j^{\prime}}=v_{j+3}$, in which case $c\left(v_{j+3} v_{j+4}\right)=x$ follows). However, applying $(*), c\left(v_{j+3} v_{j+4}\right)=x$ would imply $c\left(v_{j+1} v_{j+2}\right)=x$, contradicting the fact that $C$ is properly colored. So $c\left(v_{j+4} v_{j+5}\right)=$ $x$ holds. Similar reasoning leads to $c\left(v_{j-6} v_{j-5}\right)=x$. It follows from $(*)$ that all of $v_{j+4} v_{j+5}, v_{j+6} v_{j+7}, \ldots, v_{j-6} v_{j-5}$ are colored $x$.

Since both $v_{j+4} v_{j+5}$ and $v_{j-6} v_{j-5}$ are colored $x$, it follows from $(*)$ that $v_{j+2} v_{j+3}$ and $v_{j-4} v_{j-3}$ are also colored $x$. Combining this with the fact that $v_{j} v_{j+1}$ and $v_{j-2} v_{j-1}$ are both colored $x$, we have shown that (a) is true, which proves (11).

(12) There is an index $j, 1 \leq j \leq n$, such that $c\left(u_{2} v_{j}\right)=x$ or $c\left(u_{m-1} v_{j}\right)=y$.

Suppose $c\left(u_{2} v_{j}\right) \neq x$ and $c\left(u_{m-1} v_{j}\right) \neq y$ for all $j=1,2, \ldots, n$. By (9) and (10) we may choose $j, k \in\{1,2, \ldots, n\}$ such that $j$ satisfies (9a) and (9b), and $k$ satisfies (10a) and $(10 \mathrm{~b})$.

By (9b) we have $c\left(v_{j-2} v_{j-1}\right)=c\left(v_{j} v_{j+1}\right)=x$, from which $m \geq 4$ follows, as otherwise $u_{1} u_{2} v_{j} v_{j+1} \ldots v_{j-2} v_{j-1} u_{3}$ would be a PCHP. Now the path

$$
u_{1} v_{k-1} v_{k-2} \ldots v_{j+1} v_{j} u_{2} u_{3} \ldots u_{m-2} u_{m-1} v_{k} v_{k+1} \ldots v_{j-2} v_{j-1} u_{m}
$$

is not a PCHP, so $y=x$ follows.

We will show that (11a) holds. So suppose not; then it follows from (11) that (11b) holds. By (10b) we have $c\left(v_{k-2} v_{k-1}\right)=c\left(v_{k} v_{k+1}\right)=x$, which by (11b) implies $v_{k} \in$ $\left\{v_{j-2}, v_{j}, v_{j+2}\right\}$.

The case $v_{k}=v_{j}$ would lead to a contradiction, since the path

$$
u_{1} v_{j-2} v_{j-3} \ldots v_{j+1} v_{j} u_{2} u_{3} \ldots u_{m-2} u_{m-1} v_{j-1} u_{m}
$$

would be a PCHP.

Suppose $v_{k}=v_{j-2}$. By (10b) we then have $c\left(v_{j-4} v_{j-3}\right)=x$. Then $n \notin\{3,5\}$ follows from the fact that $C$ is properly colored, and $n \notin\{4,6\}$ holds since (11a) is not satisfied, so we deduce $n \geq 7$. Applying (4) to $s=j-3$ and $t=j$ we have $c\left(v_{j-3} v_{j}\right) \neq x$. However, the path $u_{1} v_{j-1} u_{2} u_{3} \ldots u_{m-2} u_{m-1} v_{j-2} u_{m}$ and the cycle $v_{j} v_{j+1} \ldots v_{j-4} v_{j-3} v_{j}$ are properly colored and satisfy (1), which contradicts our minimality assumption.

For $v_{k}=v_{j+2}$ we similarly conclude by (10b) and (4), with $s=j-1$ and $t=j+2$, that $c\left(v_{j-1} v_{j+2}\right) \neq x$. Examination of the path $u_{1} v_{j} u_{2} u_{3} \ldots u_{m-2} u_{m-1} v_{j+1} u_{m}$ and the 
cycle $v_{j-1} v_{j+2} v_{j+3} \ldots v_{j-2} v_{j-1}$ again leads to contradiction, which shows that (11a) does hold.

We have that $n$ is an even number, and the edges of $C$ are alternately colored $x$. Let $r \in\{1,2, \ldots, n\}$ be chosen so that $c\left(u_{3} v_{r}\right) \neq c\left(u_{3} u_{4}\right)$; this is possible by (3). We apply (5) and (6) with $p=3$ and $q=r$. Then (5d) and (6d) both fail by the choice of $r$. Neither of the edges $u_{2} v_{r-1}$ and $u_{2} v_{r+1}$ have color $x$, due to our assumption, hence (5a) and (6a) both fail. For the same reason one of (5b) and (6b) fails, because either $v_{r-2} v_{r-1}$ or $v_{r+1} v_{r+2}$ is colored $x$. If $v_{r-2} v_{r-1}$ is colored $x$, then (5b) fails, so (5c) holds and gives $c\left(u_{3} v_{r}\right)=c\left(v_{r} v_{r+1}\right)$, and now

$$
u_{1} u_{2} v_{k} v_{k+1} \ldots v_{r-1} v_{r} u_{3} u_{4} \ldots u_{m-2} u_{m-1} v_{k-1} v_{k-2} \ldots v_{r+2} v_{r+1} u_{m}
$$

is a PCHP, a contradiction. If $v_{r+1} v_{r+2}$ is colored $x$, then (6b) fails, and $c\left(u_{3} v_{r}\right)=$ $c\left(v_{r-1} v_{r}\right)$ follows similarly. But

$$
u_{1} u_{2} v_{k-1} v_{k-2} \ldots v_{r+1} v_{r} u_{3} u_{4} \ldots u_{m-2} u_{m-1} v_{k} v_{k+1} \ldots v_{r-2} v_{r-1} u_{m}
$$

is a PCHP, again with contradiction. This finishes the proof of (12).

(13) Assume $c\left(u_{2} v_{j}\right)=x$ for some $j \in\{1,2, \ldots, n\}$, and let $w=c\left(v_{j-1} v_{j}\right)$ and $z=c\left(v_{j} v_{j+1}\right)$. Then

a) $c\left(v_{j-2} v_{j-1}\right)=c\left(v_{j+1} v_{j+2}\right)=c\left(v_{j-1} v_{j+1}\right)=x$ and $n \in\{3,5\}$

b) $c\left(u_{2} v_{k}\right) \neq x$ for all $v_{k} \neq v_{j}$

c) $c\left(u_{2} v_{j-1}\right)=c\left(u_{2} v_{j+1}\right)=c\left(u_{2} u_{3}\right) \in\{w, z\}$

d) $m \geq 4$

e) if $c\left(u_{2} u_{3}\right)=w$, then $c\left(u_{3} v_{k}\right)=c\left(u_{3} u_{4}\right) \neq c\left(u_{3} v_{j-1}\right)=w$ for all $v_{k} \neq v_{j-1}$.

f) if $c\left(u_{2} u_{3}\right)=z$, then $c\left(u_{3} v_{k}\right)=c\left(u_{3} u_{4}\right) \neq c\left(u_{3} v_{j+1}\right)=z$ for all $v_{k} \neq v_{j+1}$.

We may assume $j=1$, so that $c\left(u_{2} v_{1}\right)=x, c\left(v_{n} v_{1}\right)=w$ and $c\left(v_{1} v_{2}\right)=z$. Then $c\left(u_{2} v_{1}\right)=x \neq c\left(u_{2} u_{3}\right)$ and (7b) directly imply (a). Since $n \in\{3,5\}$ and $C$ is properly colored, $c\left(u_{2} v_{k}\right)=x$ now implies $v_{k}=v_{1}$ by (a) and (7b), so (b) holds.

Now $c\left(u_{2} v_{2}\right)=c\left(u_{2} u_{3}\right)$ follows from (5) and (6) with $p=2$ and $q=2$, since (5a), (5b), (6a), (6b) all fail, and (5c) contradicts (6c), so that (5d) and the equivalent (6d) hold. Similarly we deduce $c\left(u_{2} v_{n}\right)=c\left(u_{2} u_{3}\right)$ from (5) and (6) with $p=2$ and $q=n$.

Now (d) and $c\left(u_{3} v_{1}\right)=c\left(u_{3} u_{4}\right)$ follow from (5) and (6) with $p=3$ and $q=1$, as $(5 \mathrm{~d})$ or $(6 \mathrm{~d})$ is again satisfied. Moreover for $n=5$ the identity $c\left(u_{3} v_{3}\right)=c\left(u_{3} u_{4}\right)$ 
follows, using $c\left(v_{2} v_{3}\right) \neq c\left(v_{3} v_{4}\right)$, from the fact that neither $u_{1} u_{2} v_{4} v_{5} v_{1} v_{2} v_{3} u_{3} u_{4} \ldots u_{m}$ nor $u_{1} v_{2} v_{1} u_{2} v_{5} v_{4} v_{3} u_{3} u_{4} \ldots u_{m}$ is a PCHP. Similarly we have $c\left(u_{3} v_{4}\right)=c\left(u_{3} u_{4}\right)$ when $n=5$.

We deduce $c\left(u_{3} v_{n}\right) \in\left\{w, c\left(u_{3} u_{4}\right)\right\}$, since $u_{1} v_{n-1} v_{n-2} \ldots v_{3} v_{2} u_{2} v_{1} v_{n} u_{3} u_{4} \ldots u_{m}$ is not a PCHP, Moreover $c\left(u_{3} v_{n}\right) \in\left\{c\left(u_{2} u_{3}\right), c\left(u_{3} u_{4}\right)\right\}$ follows, using $c\left(u_{2} v_{n}\right)=c\left(u_{2} u_{3}\right)$, by examining the path $u_{1} v_{n-1} v_{n-2} \ldots v_{2} v_{1} u_{2} v_{n} u_{3} u_{4} \ldots u_{m}$, and we have $c\left(u_{3} v_{n}\right) \in\left\{w, c\left(u_{3} u_{4}\right)\right\} \cap$ $\left\{c\left(u_{2} u_{3}\right), c\left(u_{3} u_{4}\right)\right\}$. A similar argument shows $c\left(u_{3} v_{2}\right) \in\left\{z, c\left(u_{3} u_{4}\right)\right\} \cap\left\{c\left(u_{2} u_{3}\right), c\left(u_{3} u_{4}\right)\right\}$. At least one of $c\left(u_{3} v_{n}\right)$ and $c\left(u_{3} v_{2}\right)$ is not equal to $c\left(u_{3} u_{4}\right)$, by (3), so it follows that either $c\left(u_{3} v_{n}\right)=c\left(u_{2} u_{3}\right)=w$ or $c\left(u_{3} v_{2}\right)=c\left(u_{2} u_{3}\right)=z$ holds. This shows the remaining parts of (c), (e) and (f), and (13) is proved.

(14) Assume $c\left(u_{m-1} v_{j}\right)=y$ for some $j \in\{1,2, \ldots, n\}$, and let $w^{\prime}=c\left(v_{j-1} v_{j}\right)$ and $z^{\prime}=c\left(v_{j} v_{j+1}\right)$. Then

a) $c\left(v_{j-2} v_{j-1}\right)=c\left(v_{j+1} v_{j+2}\right)=c\left(v_{j-1} v_{j+1}\right)=y$ and $n \in\{3,5\}$

b) $c\left(u_{m-1} v_{k}\right) \neq y$ for all $v_{k} \neq v_{j}$

c) $c\left(u_{m-1} v_{j-1}\right)=c\left(u_{m-1} v_{j+1}\right)=c\left(u_{m-2} u_{m-1}\right) \in\left\{w^{\prime}, z^{\prime}\right\}$

d) $m \geq 4$

e) if $c\left(u_{m-2} u_{m-1}\right)=w^{\prime}$, then $c\left(u_{m-2} v_{k}\right)=c\left(u_{m-3} u_{m-2}\right) \neq c\left(u_{m-2} v_{j-1}\right)=w^{\prime}$ for all $v_{k} \neq v_{j-1}$.

f) if $c\left(u_{m-2} u_{m-1}\right)=z^{\prime}$, then $c\left(u_{m-2} v_{k}\right)=c\left(u_{m-3} u_{m-2}\right) \neq c\left(u_{m-2} v_{j+1}\right)=z^{\prime}$ for all $v_{k} \neq v_{j+1}$.

(14) is proved similarly to (13).

By (12) we may assume $c\left(u_{2} v_{1}\right)=x$ without loss of generality. Let $w=c\left(v_{n} v_{1}\right)$ and $z=c\left(v_{1} v_{2}\right)$. We will further assume $c\left(u_{2} v_{n}\right)=c\left(u_{2} v_{2}\right)=c\left(u_{2} u_{3}\right)=w$, which is admissible by (13c) without loss of generality. Then $m \geq 4$ holds by (13d) and

$$
c\left(u_{3} v_{k}\right)=c\left(u_{3} u_{4}\right) \neq c\left(u_{3} v_{n}\right)=w \text { for all } v_{k} \neq v_{n}
$$

by (13e). These facts will be used frequently throughout the remaining part of the proof.

(15) $n=3$.

Suppose $n \neq 3$; from (13a) it follows that $n$ is equal to 5 . Further we then have $c\left(v_{4} v_{5}\right)=c\left(v_{2} v_{3}\right)=c\left(v_{2} v_{5}\right)=x$ by (13a), and $c\left(u_{2} v_{k}\right) \neq x$ for $v_{k} \in\left\{v_{3}, v_{4}\right\}$ by (13b). 


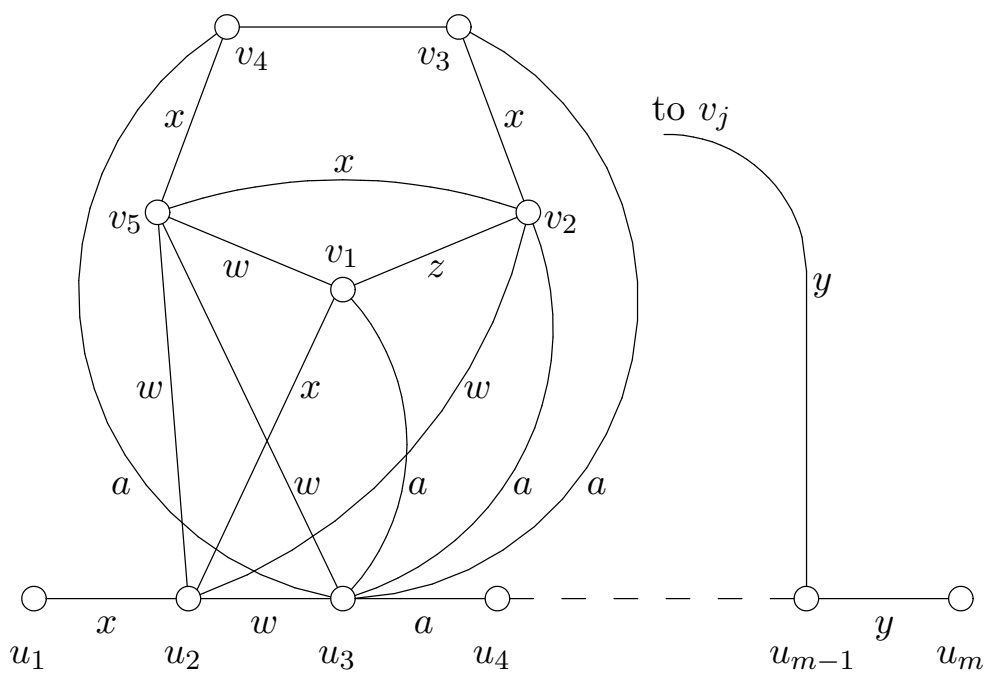

Figure 3: The situation in the proof of (15), where $a=c\left(u_{3} u_{4}\right)$.

We will first show that there exists $j \in\{1,2, \ldots, 5\}$ with $c\left(u_{m-1} v_{j}\right)=y$. So suppose not. Then by (10) there is a $k$ such that $c\left(u_{m-1} v_{k-1}\right)=c\left(v_{m-1} v_{k}\right) \neq c\left(u_{m-2} u_{m-1}\right)$ and $c\left(v_{k-2} v_{k-1}\right)=c\left(v_{k} v_{k+1}\right)=y$. The latter implies $k \notin\{1,2\}$, using $c\left(v_{4} v_{5}\right) \neq c\left(v_{1} v_{2}\right)$ and $c\left(v_{5} v_{1}\right) \neq c\left(v_{2} v_{3}\right)$. For $k \in\{3,4\}$ the path $u_{1} v_{4} u_{2} v_{1} v_{2} v_{5} u_{3} u_{4} \ldots u_{m-2} u_{m-1} v_{3} u_{m}$ is a PCHP, and for $k=5$ the path $u_{1} v_{3} u_{2} v_{1} v_{2} v_{5} u_{3} u_{4} \ldots u_{m-2} u_{m-1} v_{4} u_{m}$ is a PCHP, giving a contradiction in each case. Thus we have shown that $j$ exists as desired. Figure 3 summarizes what has been shown so far about the colors of various edges.

Now (14a) implies $y \in\{x, z, w\}$, and the value of $j$ is uniquely determined, by (14b). Moreover, (14c) implies $c\left(u_{m-2} u_{m-1}\right) \in\{x, z, w\} \backslash\{y\}$.

Case 1: $y=x$. In this case $j=1$, and $c\left(u_{m-1} v_{1}\right)=x \neq c\left(u_{m-2} u_{m-1}\right)$. However, $u_{1} u_{2} v_{2} v_{1} u_{m-1} u_{m-2} \ldots u_{4} u_{3} v_{5} v_{4} v_{3} u_{m}$ is a PCHP, a contradiction.

Case 2: $y=z$. In this case $j=5, c\left(u_{m-1} v_{5}\right)=z$, and $c\left(u_{m-2} u_{m-1}\right) \in\{x, w\}$. Supposing that $c\left(u_{m-2} u_{m-1}\right)=x$ holds, the path $u_{1} u_{2} \ldots u_{m-3} u_{m-2} v_{4} v_{3} u_{m-1} u_{m}$ and the cycle $v_{1} v_{5} v_{2} v_{1}$ would both be properly colored, contradicting our minimality assumption. So we have $c\left(u_{m-2} u_{m-1}\right)=w$.

By (13e) we have $c\left(u_{3} v_{1}\right)=c\left(u_{3} u_{4}\right)$, and $c\left(u_{m-1} v_{1}\right)=c\left(u_{m-2} u_{m-1}\right)$ follows from (14c), so we deduce $m \neq 4$. Since $c\left(u_{2} u_{3}\right)=w=c\left(u_{m-2} u_{m-1}\right)$ it is clear that $m \neq 5$ holds, hence $m \geq 6$. By $(14 \mathrm{e})$ we have $c\left(u_{m-2} v_{1}\right)=w$. Now

$$
u_{1} u_{2} v_{2} v_{1} u_{m-2} u_{m-3} \ldots u_{4} u_{3} v_{5} v_{4} v_{3} u_{m-1} u_{m}
$$




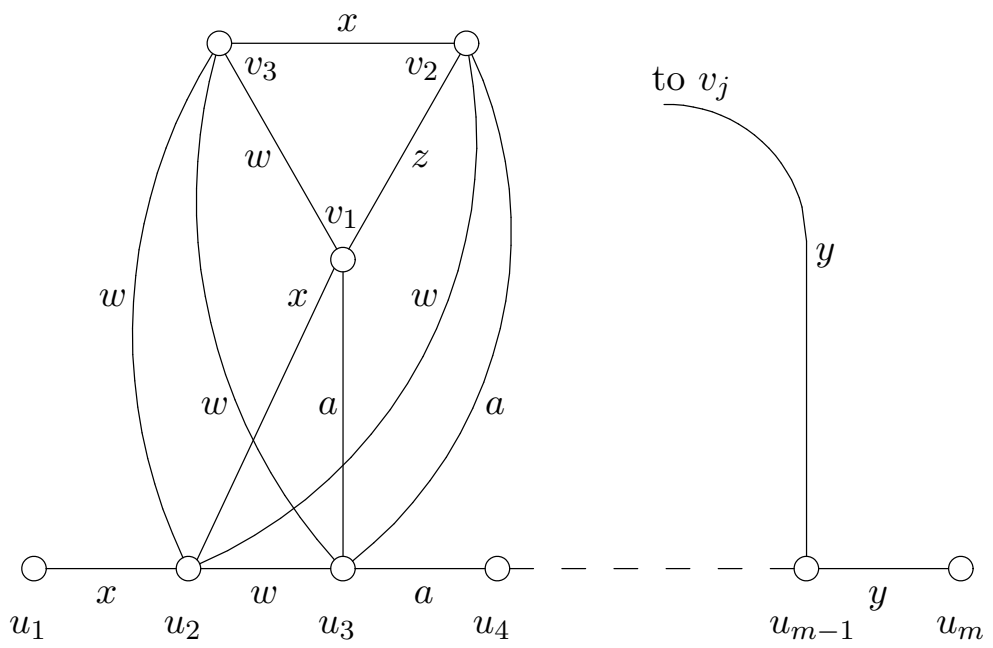

Figure 4: The situation in the final steps of the proof of Claim A, where $a=c\left(u_{3} u_{4}\right)$.

is a PCHP, a contradiction.

Case 3: $y=w$. In this case $j=2$, and $c\left(u_{m-1} v_{2}\right)=w$. We note by (14a) that $c\left(v_{1} v_{3}\right)=w$ holds. However $u_{1} u_{2} v_{4} v_{5} u_{3} u_{4} \ldots u_{m}$ and $v_{1} v_{2} v_{3} v_{1}$ are now properly colored, again contradicting our minimality assumption. This finishes the last case, and (15) is proved.

We choose $j \in\{1,2,3\}$ with $c\left(u_{m-1} v_{j}\right)=y$, which is possible since (10b) fails for $n=3$, implying that the assumption of (10) does not hold. Then $j$ satisfies the assumption of (8), hence $c\left(v_{j+1} v_{j+2}\right)=y$ follows from (8b). We deduce $y \in\{x, z, w\}$ and proceed to divide into the three respective cases (see also Figure 4).

Case 1: $y=x$. Then $j=1$ follows from $c\left(v_{2} v_{3}\right)=x$, and the choice of $j$ implies $c\left(u_{m-1} v_{1}\right)=x$. But now $u_{1} u_{2} v_{2} v_{1} u_{m-1} u_{m-2} \ldots u_{4} u_{3} v_{3} u_{m}$ is a PCHP, a contradiction.

Case 2: $y=z$. In this case $j=3$ follows from $c\left(v_{1} v_{2}\right)=y$, implying $c\left(u_{m-1} v_{3}\right)=z$. By $(13 \mathrm{e}) c\left(u_{3} v_{1}\right)=c\left(u_{3} v_{2}\right)=c\left(u_{3} u_{4}\right) \neq c\left(u_{3} v_{3}\right)=w$. For $m=4$ all of $(6 \mathrm{a}, \mathrm{b}, \mathrm{c}, \mathrm{d})$ would fail for $p=4$ and $q=1$ (in particular (6b) fails by $c\left(u_{3} v_{2}\right)=c\left(u_{3} u_{4}\right)=y \neq x=c\left(v_{2} v_{3}\right)$ ), so we deduce $m \geq 5$.

Suppose $c\left(u_{4} v_{1}\right) \neq c\left(u_{4} u_{5}\right)$. Then $c\left(u_{3} v_{1}\right)=c\left(u_{4} v_{1}\right)$ holds, as otherwise the path $u_{1} u_{2} v_{2} v_{3} u_{3} v_{1} u_{4} u_{5} \ldots u_{m}$ would be a PCHP. Now (6) with $p=4$ and $q=1$ implies $c\left(u_{3} v_{2}\right)=x$ or $c\left(u_{4} v_{1}\right)=w$ (i.e. (6b) or $\left.(6 \mathrm{c})\right)$. We have $c\left(u_{4} v_{1}\right)=c\left(u_{3} v_{1}\right)=c\left(u_{3} u_{4}\right) \neq$ 
$c\left(u_{2} u_{3}\right)=w$, so $c\left(u_{3} v_{2}\right)=x$ follows. But then $u_{1} u_{2} v_{2} u_{3} v_{3} u_{m-1} u_{m-2} \ldots u_{5} u_{4} v_{1} u_{m}$ is a PCHP, which is a contradiction. We conclude that $c\left(u_{4} v_{1}\right)=c\left(u_{4} u_{5}\right)$ holds.

Suppose $c\left(u_{4} v_{2}\right) \neq c\left(u_{4} u_{5}\right)$. Then (5c) for $p=4$ and $q=2$ holds, that is $c\left(u_{4} v_{2}\right)=x$, and $u_{1} u_{2} u_{3} v_{1} v_{3} u_{m-1} u_{m-2} \ldots u_{5} u_{4} v_{2} u_{m}$ is a PCHP, a contradiction. Hence $c\left(u_{4} v_{2}\right)=$ $c\left(u_{4} u_{5}\right)$ follows. Now by (3) we have $c\left(u_{4} v_{3}\right) \neq c\left(u_{4} u_{5}\right)$. With $p=4$ and $q=3$ either $(5 \mathrm{~b})$ or $(5 \mathrm{c})$ holds, hence $c\left(u_{3} v_{2}\right)=z$ or $c\left(u_{4} v_{3}\right)=w$. Examination of the path $u_{1} v_{2} u_{2} v_{1} u_{3} v_{3} u_{4} u_{5} \ldots u_{m}$ allows us to deduce $c\left(u_{3} v_{1}\right)=x$ or $c\left(u_{4} v_{3}\right)=w$. Then $c\left(u_{4} v_{3}\right)=w$ follows, since the two alternatives conflict due to $c\left(u_{3} v_{1}\right)=c\left(u_{3} v_{2}\right)$.

Let $i$ be the largest number such that $i \leq m$ and such that $c\left(u_{i^{\prime}} v_{1}\right)=c\left(u_{i^{\prime}} v_{2}\right)=$ $c\left(u_{i^{\prime}} u_{i^{\prime}+1}\right) \neq c\left(u_{i^{\prime}} v_{3}\right)=w$ holds for every $i^{\prime}=3,4, \ldots, i-1$. We note that $i$ exists and satisfies $i \geq 5$. It is useful to observe that (5a) and (6a) fail for $p=i$ and every $q=1,2,3$; for $(5 \mathrm{a})$ this follows from $c\left(u_{i-2} u_{i-1}\right) \neq w$ for $q=1$, and it follows from $c\left(u_{i-1} v_{q-1}\right)=c\left(u_{i-1} u_{i}\right) \neq c\left(u_{i-2} u_{i-1}\right)$ for $q \neq 1$. Similarly for $(6 \mathrm{a})$.

For $i=m$ all of $(6 \mathrm{a}, \mathrm{b}, \mathrm{c}, \mathrm{d})$ fail for $p=i$ and $q=1$ (in particular (6b) fails by $\left.c\left(u_{i-1} v_{2}\right)=c\left(u_{i-1} u_{i}\right)=y \neq x=c\left(v_{2} v_{3}\right)\right)$, so we deduce $i<m$.

Suppose $c\left(u_{i} v_{1}\right) \neq c\left(u_{i} u_{i+1}\right)$. For $p=i$ and $q=1$ (5) and (6) imply $c\left(u_{i} v_{1}\right)=z$ and $c\left(u_{i-1} v_{2}\right)=x$ (respectively $(5 \mathrm{c})$ and $\left.(6 \mathrm{~b})\right)$. Using $c\left(u_{i-1} v_{1}\right)=c\left(u_{i-1} v_{2}\right)=x$ and $c\left(u_{i-2} v_{2}\right)=c\left(u_{i-2} u_{i-1}\right) \neq c\left(u_{i-1} u_{i}\right)=c\left(u_{i-1} v_{2}\right)=x$, the path

$$
u_{1} \ldots u_{i-3} u_{i-2} v_{2} v_{3} u_{i-1} v_{1} u_{i} u_{i+1} \ldots u_{m}
$$

is a PCHP. This contradiction shows $c\left(u_{i} v_{1}\right)=c\left(u_{i} u_{i+1}\right)$. Suppose $c\left(u_{i} v_{2}\right) \neq c\left(u_{i} u_{i+1}\right)$. Then (5) with $p=i$ and $q=2$ implies $c\left(u_{i} v_{2}\right)=x$, but

$$
u_{1} u_{2} \ldots u_{i-2} u_{i-1} v_{1} v_{3} u_{m-1} u_{m-2} \ldots u_{i+1} u_{i} v_{2} u_{m}
$$

is a PCHP, a contradiction. Therefore $c\left(u_{i} v_{2}\right)=c\left(u_{i} u_{i+1}\right)$. By (3) we have $c\left(u_{i} v_{3}\right) \neq$ $c\left(u_{i} u_{i+1}\right)$. Then $c\left(u_{i} v_{3}\right) \neq w$ follows from the choice of $i$, and

$$
u_{1} v_{2} v_{1} u_{2} u_{3} \ldots u_{i-2} u_{i-1} v_{3} u_{i} u_{i+1} \ldots u_{m}
$$

is a PCHP, a contradiction.

Case 3: $y=w$. This implies $j=2$ and $c\left(u_{m-1} v_{2}\right)=w$. By (14c) we have $c\left(u_{m-2} u_{m-1}\right) \in\{x, z\}$. The case $c\left(u_{m-2} u_{m-1}\right)=z$ is symmetric to Case 2 , so only the case $c\left(u_{m-2} u_{m-1}\right)=x$ remains. Then $m \geq 5$ follows, as $P$ is properly colored. We can assume $c\left(u_{2} u_{m-1}\right) \neq x$ without loss of generality. But $u_{1} u_{2} u_{m-1} u_{m-2} \ldots u_{4} u_{3} v_{3} v_{2} v_{1} u_{m}$ is a PCHP, with contradiction. This finishes the proof of Case 3, and of Claim A. 


\section{References}

[1] N. Alon and G. Gutin, Properly colored Hamilton cycles in edge colored complete graphs. Random Structures and Algorithms 11 (1997) 179-186.

[2] J. Bang-Jensen and G. Gutin, Alternating cycles and paths in edge-coloured multigraphs: a survey. Discrete Math. 165/166 (1997) 39-60.

[3] J. Bang-Jensen and G. Gutin, Digraphs: Theory, Algorithms and Applications, Springer-Verlag, London, 2000.

[4] J. Bang-Jensen, G. Gutin and A. Yeo, A polynomial algorithm for the Hamiltonian cycle problem in semicomplete multipartite digraphs. J. Graph Theory 29 (1998) 111132.

[5] J. Bang-Jensen, G. Gutin and A. Yeo, Properly coloured Hamiltonian paths in edgecoloured complete graphs. Discrete Appl. Math. 82 (1998) 247-250.

[6] M. Bankfalvi and Zs. Bankfalvi, Alternating hamiltonian circuit in two-coloured complete graphs, (Proc. Colloq. Tihany 1968), Academic Press, New York (1968) 11-18.

[7] O. Barr, Properly coloured Hamiltonian paths in edge-coloured complete graphs without monochromatic triangles. Ars Combinatoria 50 (1998) 316-318.

[8] A. Benkouar, Y. Manoussakis, V. Paschos and R. Saad, On the complexity of finding alternating Hamiltonian and Eulerian cycles in edge-coloured graphs. Lecture Notes in Comput. Sci., 557 (Springer, Berlin, 1991) 190-198.

[9] B. Bollobás and P. Erdős, Alternating Hamiltonian cycles. Israel J. Math. 23 (1976) 126-131.

[10] G. Gutin, Finding a longest path in a complete multipartite digraph. SIAM J. Discrete Math. 6 (1993) 270-273

[11] R. Saad, Finding a longest alternating cycle in a 2-edge-coloured complete graph is in RP. Combinatorics, Probability and Computing 5 (1996) 297-306.

[12] J. Shearer, A property of the colored complete graph. Discrete Math. 25 (1979) 175178. 